\title{
Preservación alveolar post extracción en zona estética: Decisiones clinicas predecibles en sitio severamente afectado.
}

\section{Alveolar ridge preservation in the esthesic zone: predictable decisions in a severly affected site.}

\begin{abstract}
Paulina Oddó ${ }^{1}$, Catherine Klein ${ }^{1 *}$, Andrés Contreras ${ }^{2}$
1. Departamento de Periodoncia, Universidad del Desarrollo-Clínica Alemana. Escuela de Odontología, Facultad de Medicina CAS-UDD, Santiago, Chile.

2. Docente del Departamento de Periodoncia, Universidad del Desarrollo- Clínica Alemana. Escuela de Odontología, Facultad de Medicina CAS-UDD, Santiago, Chile.

* Correspondencia Autor: Catherine Klein Riquelme | Dirección: Av. El Bosque 1080 departamento 303, Providencia-Santiago de Chile. | E-mail: cathykleinr@gmail.com. | Código postal: 7511300. | Teléfono: +569 84292641

Trabajo recibido el 11/06/2019.

Aprobado para su publicación el 04/02/2020

RESUMEN

Introducción: La valoración particular de sitios severamente comprometidos, involucra considerar los tiempos necesarios de cicatrización, así como evidencia actual en términos de biomateriales y técnicas quirúrgicas con el fin de lograr un tratamiento exitoso. Material y método: Paciente sexo femenino, 28 años, asiste por dolor e infección en diente 2.1 al Postítulo de Periodoncia UDD. Se observa defecto extenso y lesión que compromete tanto las tablas óseas vestibular como palatina. El tratamiento consistió en: exodoncia y regeneración ósea, instalación del implante 6 meses después de la exodoncia y cirugía de conexión 7 meses después más injerto de tejidos blandos. Resultados: El tratamiento de defectos combinados (tejidos duros y blandos), asociados a procesos infecciosos de larga data, mediante rehabilitación implanto soportada puede ser muy predecible y exitoso en la medida que se respeten los tiempos de regeneración de diferentes estructuras.
\end{abstract}

PALABRAS CLAVE:

Implante tardío; Zona estética; Regeneración ósea guiada.

International Journal of Interdisciplinary Dentistry Vol. 13(1); 30-34, 2020.

\section{ABSTRACT}

Introduction: The specific assessment of a severely compromised sites involves: the consideration of healing time according to the different kinds of tissues involved and the knowledge of the evidence available concerning biomaterials and surgical techniques. Material and methods: Female patient attends the postgraduate school of periodontics, UDD University in Santiago de Chile, because of pain and chronic infection compromising tooth 2.1. At clinical evaluation, the site has an extensive defect, with active fistula that compromises the buccal and palatal bone plates. The treatment consisted of exodontia and guided bone regeneration, implantation six months after initial exodontia and abutment connection surgery seven months after implant insertion. Results: the treatment of combined defects associated with a long-standing infectious process can be very predictable and successful, only if the measures of time and tissue handling are considered and applied.

KEY WORDS:

Late implant placement; Esthetic zone; Guided tissue regeneration; Osseous defects.

International Journal of Interdisciplinary Dentistry Vol. 13(1); 30-34, 2020.

\section{INTRODUCCIÓN:}

La oseointegración de un implante, la correcta posición tridimensional de éste, y un contorno de tejidos duros y blandos adecuados son factores muy importantes para el éxito de tratamiento. Para ello, se requiere un volumen de tejido óseo suficiente y una arquitectura favorable del vano en conjunto con una técnica quirúrgica apropiada ${ }^{(1)}$.

La predictibilidad de un tratamiento en el sector anterior estético depende de las características del tejido (duros y blandos) al momento del comienzo del tratamiento. Así, los desafíos clínicos aumentan cuando nos encontramos ante un escenario adverso de gran falta de ambas estructuras.

Hoy en día, los implantes unitarios tienen un alto grado de predictibilidad, dado que los dientes adyacentes proveen una subestructura morfológica que es necesaria para restaurar una arquitectura gingival natural ${ }^{(2-3)}$.

Los criterios de éxito de Albrektsson han sido expandido por otros autores (Smith y Zarb), considerando que un implante no sólo es exitoso si es funcional, asintomático y estable desde su oseointegración, sino que además provee una rehabilitación estética adecuada ${ }^{(4)}$.

Para lograr un resultado estéticamente óptimo, el implante se debe ubicar en una posición e inclinación adecuadas. Idealmente, una rehabilitación implanto-soportada estética imita en apariencia la del diente natural vecino. El nivel de los tejidos peri-implantarios, que influye en la altura de la corona, así como su color y textura son decisivos en la apariencia "natural" de la rehabilitación.

Hoy en día existen varias escalas de evaluación desarrolladas para la valoración estética de la rehabilitación implanto-soportada, entre ellas están el PES (Pink Esthetic Score) y el WES (White Esthetic Score). 
Ambos son índices confiables para evaluar los resultados estéticos. El índice estético rosa constituye una puntuación reproducible y utilizada ampliamente en la bibliografía. Sin embargo, ha de mencionarse el inconveniente de que el PES sólo puede aplicarse a implantes dentales unitarios y únicamente si se dispone del diente de referencia.

La extracción de un diente genera una serie de eventos biológicos, mediados tanto por una respuesta inflamatoria local posterior a la cirugía como por la ausencia de estímulo masticatorio a través del periodonto. Estos eventos gatillan una alteración de la homeostasis e integridad estructural del periodonto. Como consecuencia, en las primeras semanas se produce una atrofia del tejido, caracterizada por una reabsorción considerable del hueso alveolar y una invaginación parcial de la mucosa. La extensión y magnitud del proceso de remodelación ósea va a variar dependiendo de factores locales y/o sistémicos, pero normalmente resulta en una reducción tanto vertical como horizontal que afecta en mayor medida la porción vestibular del sitio $^{(4)}$.

Es por lo anterior, que en un intento de atenuar los eventos reabsortivos posteriores a la exodoncia de un diente, se han propuesto diferentes alternativas, incluyendo protocolos de exodoncia parcial(5), extrusión ortodóntica y preservación alveolar.

El concepto de preservación alveolar tiene relación con cualquier procedimiento realizado al momento de la exodoncia de un diente y que está diseñado para minimizar la reabsorción externa del reborde y maximizar la formación ósea dentro del alvéolo.

Existen diferentes técnicas de preservación alveolar. Algunos incluyen el uso de injertos óseos, membranas de barrera o una combinación de ambos. En general, las terapias de preservación alveolar son procedimientos con enfoque regenerativo de naturaleza interceptiva de comprobada efectividad como se ha descrito en diferentes revisiones sistemáticas ${ }^{(6)}$.

El desafío de lograr una terapéutica exitosa aumenta, cuando el sitio a intervenir se encuentra en el sector anterior del maxilar. Esta zona tiene alta demanda estética, por lo que normalmente se realizan procedimientos de aumento de tejido tanto óseo como blando y se proveen mayores tiempos de estabilización del sitio (4-6 meses) previo a la instalación del implante. En algunos casos, la instalación de un implante inmediato es factible, sin embargo, debe cumplir con requisitos específicos para hacer predecible el tratamiento ${ }^{(7)}$.

El objetivo de este reporte es discutir el paso a paso de un escenario clínico altamente complejo en el sector anterior estético y la predictibilidad de los resultados alcanzados.

\section{MATERIAL Y MÉTODO}

Paciente género femenino, 28 años, ASA II (Insulino resistente controlada) acude al postítulo de Periodoncia de la Universidad del Desarrollo y consulta por dolor e infección en diente 2.1 con historia previa de trauma en la infancia.

Se observa diente 2.1 con cambio de coloración, tratado endodónticamente hace 17 años con fractura radicular vertical con desplazamiento; fístula vestibular activa con fenestración ósea vestibular (3 $\mathrm{mm}$ aproximadamente) y Palatina $(4 \mathrm{~mm}$ ). El CBCT (cone beam computed tomography) muestra además de la pérdida ósea vestibular y palatina, una solución de continuidad que se extiende muy cerca del reborde infraorbitario. Al momento del examen se realiza una valoración PES de 5 puntos.

En una primera etapa, se planificó la exodoncia y regeneración ósea guiada previo a la instalación del implante. Para las incisiones, se utilizó un bisturí hoja 15c mango redondo, con incisión intrasulcular que se extendió un diente a cada lado del defecto. La incisión vertical se ubicó en la línea angular distal de cada diente en posición para mediana. EI diseño de colgajo se basó en la magnitud del defecto y el compromiso del aspecto palatino. Se elevó un colgajo a espesor total vestibular y palatino; posteriormente a esto se realizó la exodoncia atraumática del diente 2.1 con periótomo, se limpió y eliminó todo el tejido de granulación en relación con el defecto óseo. Se evaluó el tamaño del defecto (20 $\mathrm{mm}$. x $10 \mathrm{~mm}$.) y se rellenó con $1 \mathrm{cc}$. de injerto particulado Puros cortical $\left(\right.$ Zimmer $\left.^{\circledR}\right)$, cubierto por una membrana de colágeno Jason Botiss Pericardio $\left(\right.$ Botiss $\left.^{\circledR}\right)$. Para el cierre de la herida, se realizó una incisión liberadora en el periostio para permitir el cierre sin tensión de ésta. Se suturó mediante puntos simples con nylon 5.0. A las dos semanas se evidenció una dehiscencia del colgajo en la parte media del aspecto vestibular de la incisión supracrestal, por lo que se pasó un nuevo punto de sutura nylon 5.0 en la zona expuesta. Inmediatamente posterior a la cirugía, la paciente se mantuvo con un provisorio oval en base a una prótesis parcial removible de transición.

A los 6 meses (8 de agosto 2018), se toma un nuevo CBCT de la zona intervenida y se planificó la cirugía de colocación del implante. El estudio complementario previo del CBCT y el posterior acceso al sitio quirúrgico, permiten determinar la gran cantidad de tejido óseo disponible para la instalación adecuada del implante, considerando que esta ganancia fue a expensas de un hueso de calidad tipo 4. Para la implantación, se realizó el protocolo de fresado de acuerdo con las indicaciones del fabricante y se instaló un implante MIS SEVEN de $3,3 \mathrm{~mm} \times 11,5 \mathrm{~mm}$, logrando un torque de $30 \mathrm{nM}$. Posteriormente se cerró el colgajo por primera intención y la paciente se mantuvo en controles sucesivos sin inconvenientes.

Después de 7 meses, se planificó la cirugía de conexión del implante, donde además se realizó un aumento de tejidos blandos mediante injerto conectivo palatino subepitelial, con el objetivo de mejorar el contorno de tejido blando vestibular. Posterior a la cirugía, el implante quedó con un pilar temporal (MIS Pilar Temporal Peek $ø 4 \mathrm{~mm}$ ) y provisorio en el cual se trabajó el perfil de emergencia definiendo áreas crítica y subcrítica para la conformación de concavidad y convexidad. La restauración definitiva del implante se terminó dos meses después de la cirugía de conexión. El implante lleva actualmente 3 meses sometido a carga. De acuerdo con el último registro fotográfico se logró un registro PES de 9 puntos.

\section{DISCUSIÓN}

La toma de decisiones hoy en día en relación con el plan de tratamiento de un diente con indicación de extracción debe realizarse previo a la exodoncia de éste. Existen diferentes modalidades de tratamiento, dependiendo del objetivo a lograr (Preservación de tejidos duros, blandos o ambas). En este caso, el enfoque fue la regeneración ósea guiada de un defecto completo en ancho y altura (más una fenestración vestibular y otra palatina), que comprometía más del $50 \%$ de estas estructuras y la planificación posterior de un implante tardío (Mayor a 6 meses) ${ }^{(7)}$. Debido a que los cambios dimensionales del vano posteriores a la exodoncia son inevitables e irreversibles, es que se han propuesto diversas técnicas de preservación alveolar. Si bien el objetivo no es evitar este fenómeno biológico, el uso de distintos biomateriales (sustitutos óseos con o sin membrana de barrera) puede reducir el grado de alteraciones dimensionales ${ }^{(8)}$.

La preservación alveolar será indicada cuando, por razones del paciente (edad, compromiso sistémico, factores económicos) o del sitio (defecto óseo muy extenso, déficit de tejidos blandos) no se pueda instalar un implante inmediato o temprano. El objetivo en este caso, relacionado con el sitio, fue lograr un volumen óseo tridimensional adecuado del vano desdentado, incluyendo una pared ósea vestibular de suficiente altura y grosor, para lograr instalar un implante en su posición protésica ideal.

El uso de biomateriales de injerto en el alvéolo ha ganado popularidad en el último tiempo. Así, se han desarrollado una vasta cantidad de materiales incluyendo: Hueso autólogo, aloinjertos, xenoinjertos, aloplastos, derivados plaquetarios y agentes bioactivos ${ }^{(8)}$. En la conferencia consenso de Osteology Foundation (2012), así como en estudios más recientes ${ }^{(9)}$, la mayoría de los estudios y revisiones sistemáticas no revelaron ninguna superioridad entre un material y otro, pero sí recalcaron la importancia del cierre primario de la herida. Una revisión sistemática mostró, que el cierre primario de la herida, uso de membrana y xenoinjerto o aloinjerto se asoció con resultados más exitosos, particularmente en relación con preservación en altura de las porciones medio-vestibular y medio-palatina del proceso alveolar.

El manejo de tejidos blandos es esencial en procedimientos regenerativos, sobre todo si el sitio se encuentra en zona estética. Desde un punto de vista estético y preservación de vascularización, se sugiere el diseño de un colgajo con una sola incisión de descarga, distal al canino o incluso al primer premolar; Sin embargo, si no es posible por el tamaño del defecto o por el largo del vano, se puede elegir un colgajo trapezoidal que permita un cierre sin tensión del colgajo(10).

El estudio de Tonetti(11) reportó resultados interesantes al comparar 124 pacientes operados ya sea mediante implante inmediato post extracción o 3 meses después. En sus estudios describen que, si bien la cirugía de implante inmediato disminuye la comorbilidad y tiempos de tratamiento, no está indicado en todos los casos. La instalación inmediata de implantes conlleva mayor prevalencia de falla en el cierre de la herida (5 veces mayor), mayor necesidad de sustitutos óseos, mayor tasa de resultados estéticos poco óptimos y comparativamente una ubicación más apical del implante, con mayor profundidad al sondaje y una tendencia a mayor pérdida ósea radiográfica.

Se discute, además, que en varios casos $(7,5 \%$ de los casos en este estudio) el alveolo no estaba en condiciones para la instalación de un implante inmediato. Probablemente, dichos casos se vean afectados en alguno de los factores relacionados con el éxito de la terapia. Estos eventos, reportados ampliamente en la literatura se asocian a una falta frecuente de valoración del clínico de diagnóstico del sitio y por otro lado conciencia, que no todos los sitios han de tener la misma sistemática. 
Desde un punto de vista de dificultad, Tonetti et al.(11) describieron que ambos procedimientos quirúrgicos presentaron desafíos específicos. En términos de resultados relacionados con el paciente no se han reportado diferencias en términos de dolor o disconfort. Otros estudios, describen una tendencia a mayor incomodidad postquirúrgica en las etapas iniciales de cicatrización en implantes inmediatos.

Basado en este estudio clínico randomizado y considerando las características del sitio comprometido en la paciente, es que lo más indicado fue un protocolo diferido de instalación del implante, con el fin de optimizar los resultados estéticos y funcionales.

\section{CONCLUSIÓN}

Los resultados de este reporte muestran que la rehabilitación estética exitosa implanto-soportada en el sector anterior de un sitio severamente comprometido es posible, en la medida que el clínico integre y aplique conocimientos tanto de técnicas quirúrgicas y biomateriales como de tiempos en relación con la cicatrización de los tejidos. Si bien por consideraciones de tiempo y morbilidad se elige comúnmente un enfoque de implante inmediato o temprano, en algunos casos el escenario imposibilita esas posibilidades. La técnica de preservación alveolar es efectiva en el manejo de defectos de gran magnitud y el uso de aloinjertos

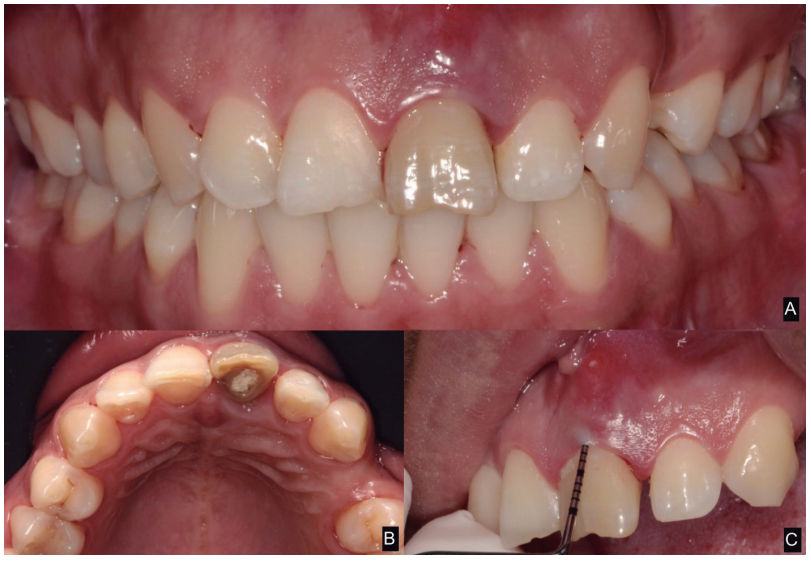

Figura 1. Condición inicial

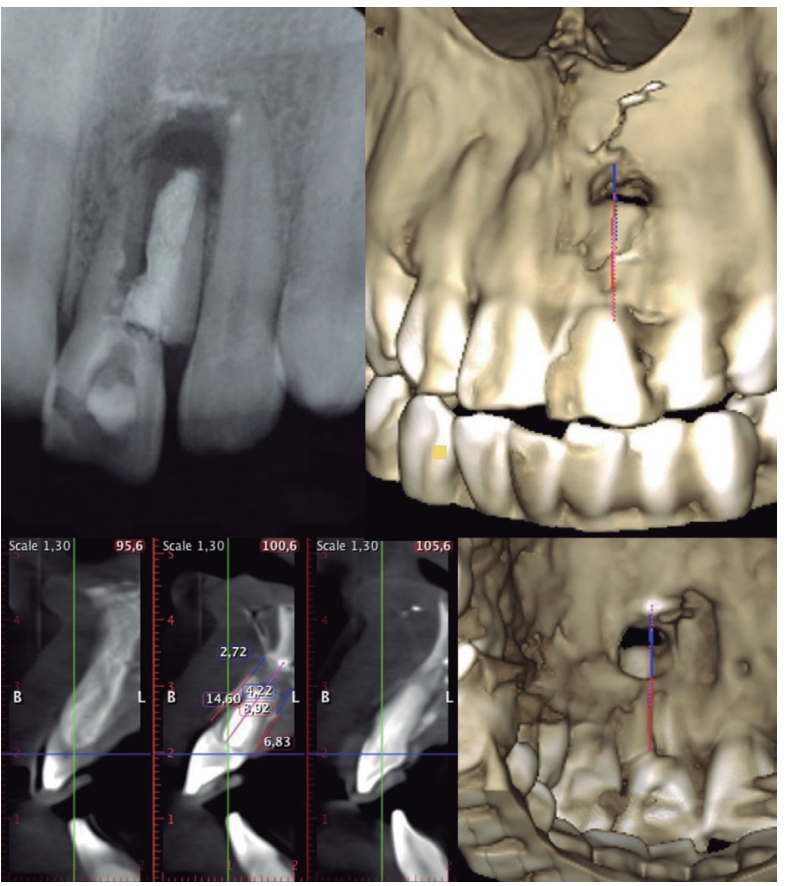

Figura 2. Evaluación CBCT sitio afectado provee un continente suficiente para la estabilización adecuada del implante.

Por otro lado, técnicas combinadas con injerto de tejido óseo y blando en tiempos diferidos a la instalación de implante inmediato permite preservar y devolver contornos adecuados en pacientes con pérdida previa de tejido óseo tanto en biotipos fino como grueso.

La secuencia de tratamiento realizada en la paciente permitió compensar la inevitable reabsorción y remodelación tanto del hueso remanente como del injerto óseo, evitando así alteraciones en los tejidos peri-implantarios. Más aún, el uso de injerto de tejido conectivo permitió mejorar el perfil y arquitectura de tejidos blandos, en un escenario con claras secuelas de enfermedad, logrando una rehabilitación altamente estética y satisfactoria.

\section{CONFLICTO DE INTERESES}

Declaramos no tener ningún tipo de conflicto de intereses, ni ninguna relación económica, personal, política, interés financiero ni académico que pueda influir en nuestro juicio. Declaramos, además, no haber recibido ningún tipo de beneficio monetario, bienes ni subsidios de alguna fuente que pudiera tener interés en los resultados de esta investigación.

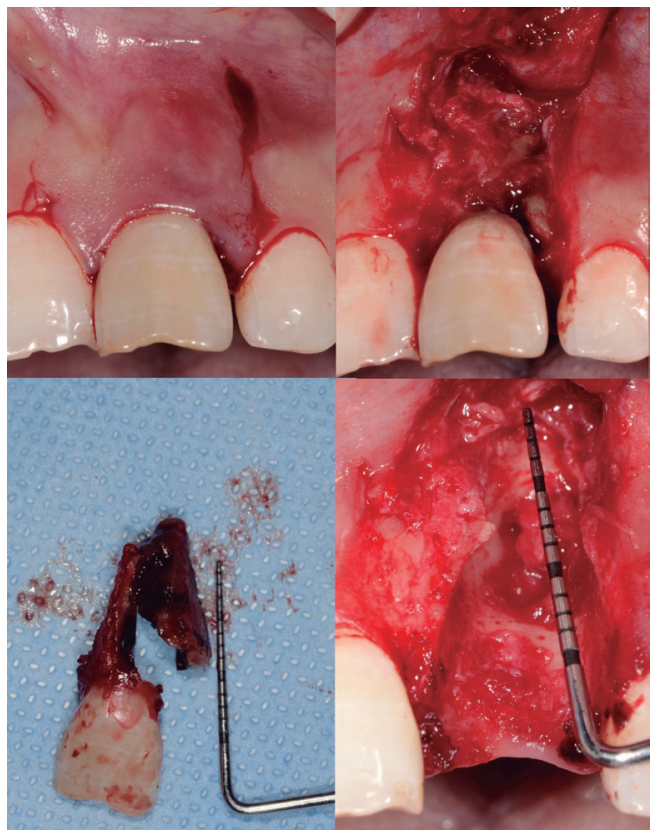

Figura 3. Exodoncia y valoración quirúrgica del defecto.

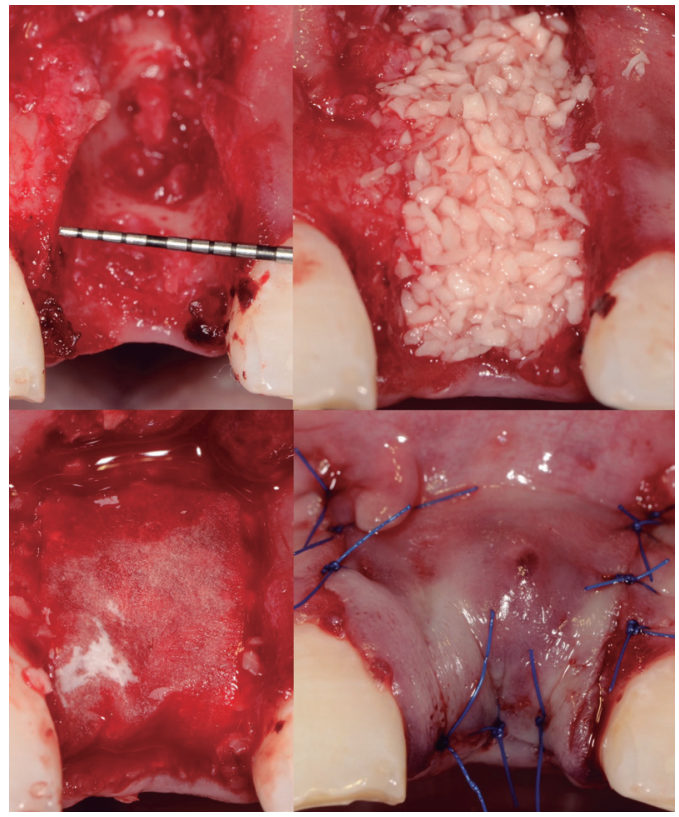

Figura 4. Regeneración ósea guiada 


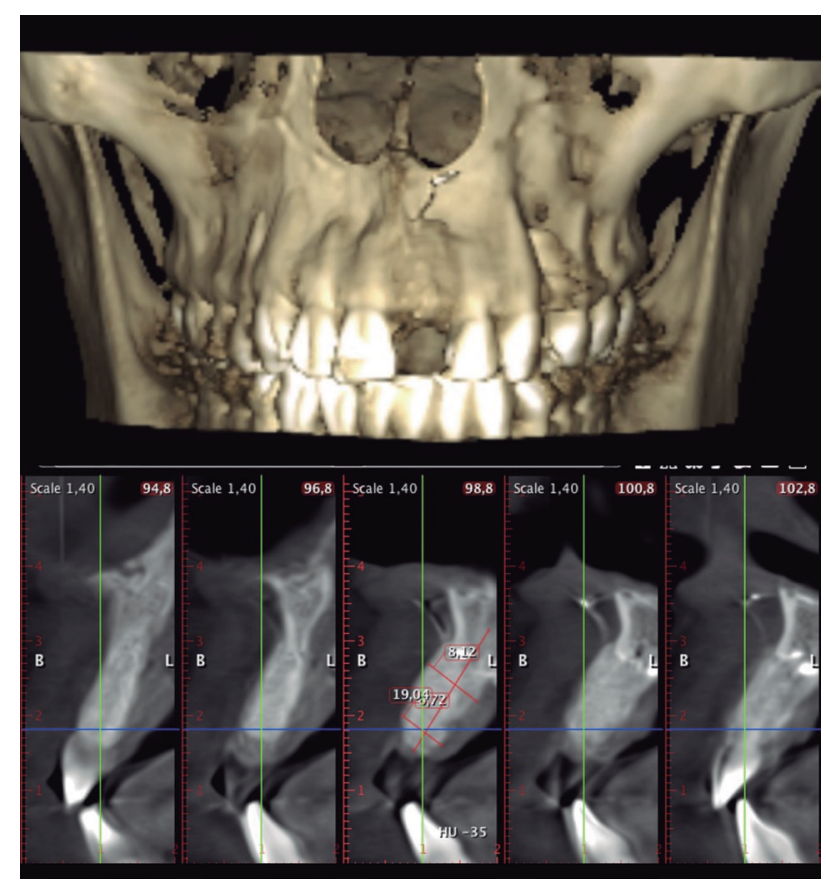

Figura 5. CBCT 6 meses post quirúrgico

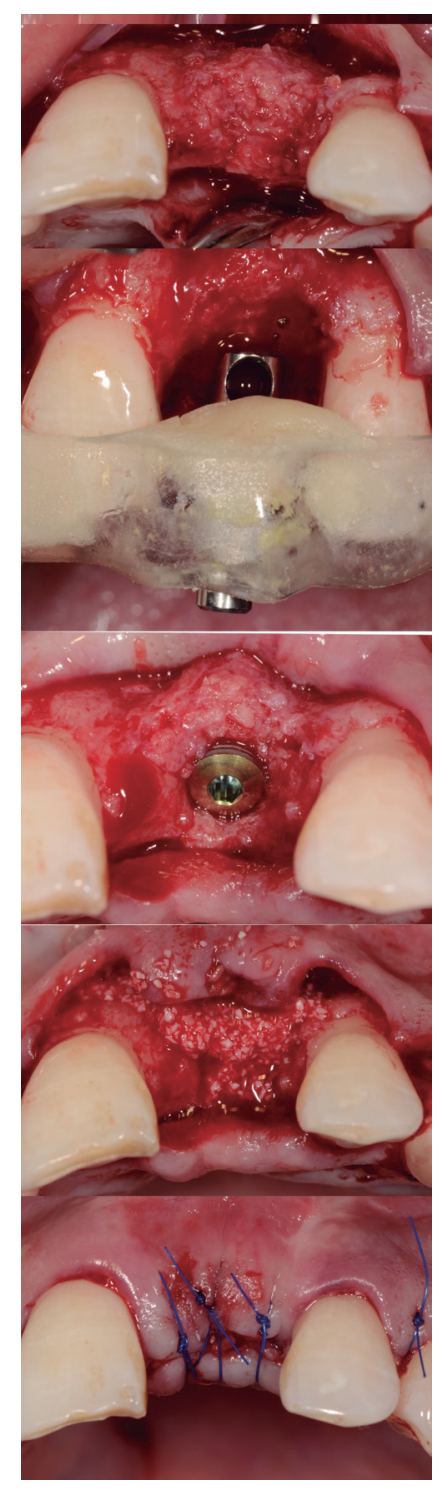

Figura 6. Instalación del Implante

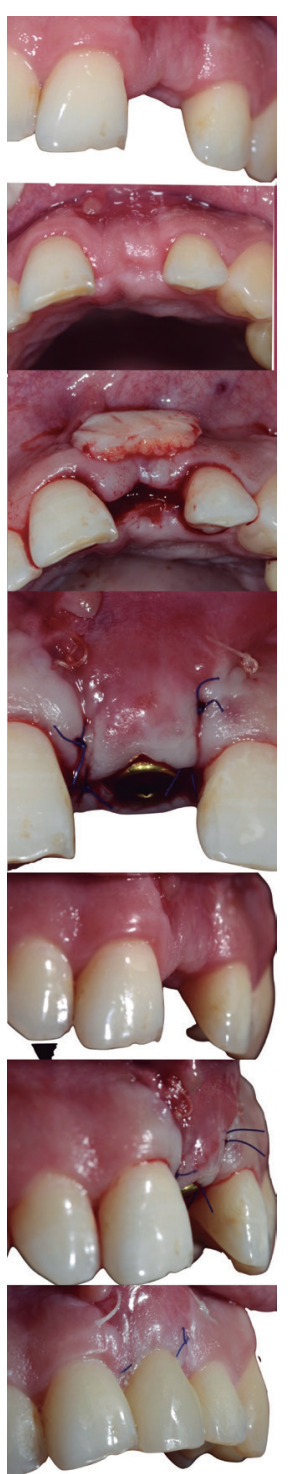

Figura 7. Cirugía de conexión y manejo de tejidos blandos

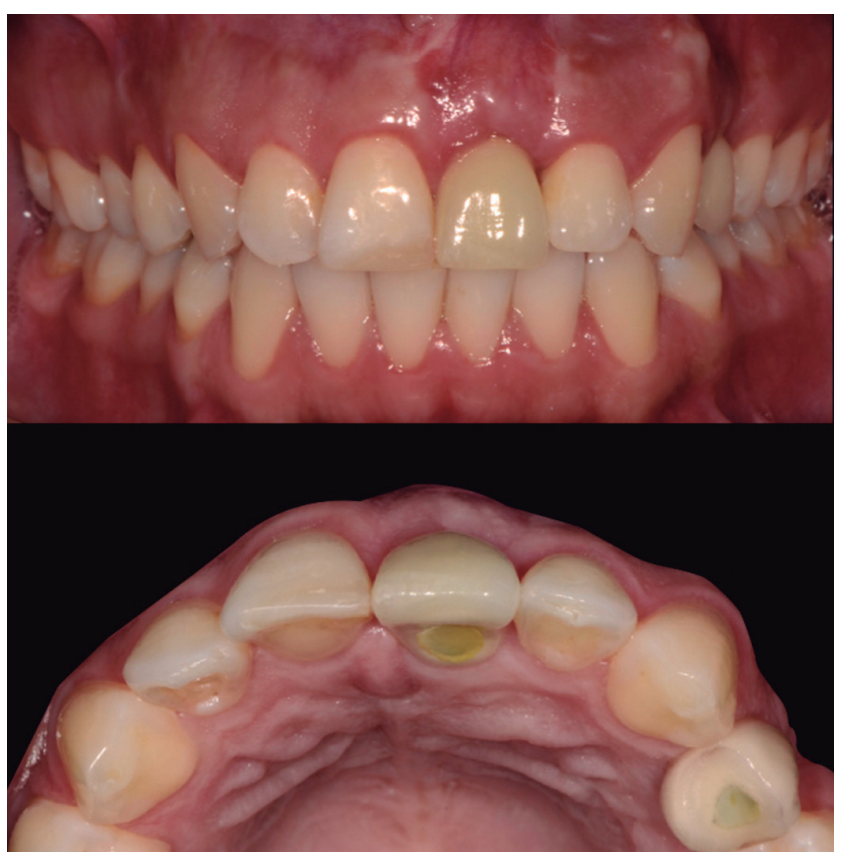

Figura 8. 


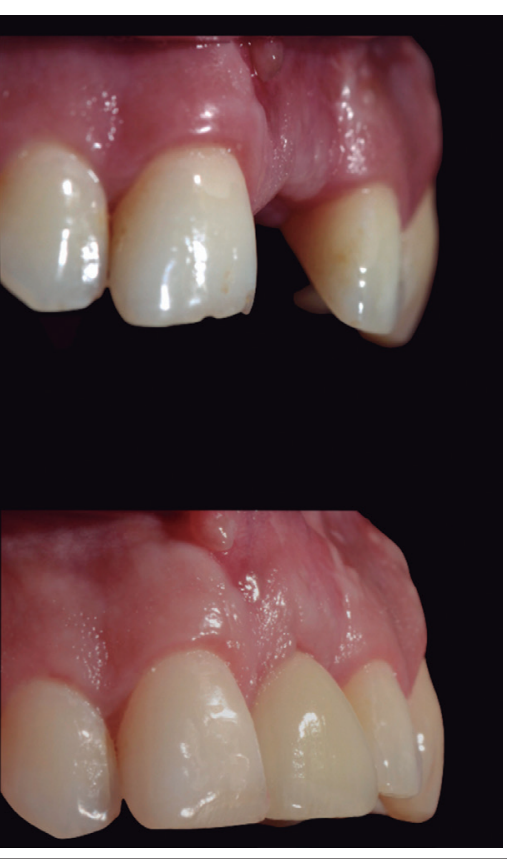

Figura 9.

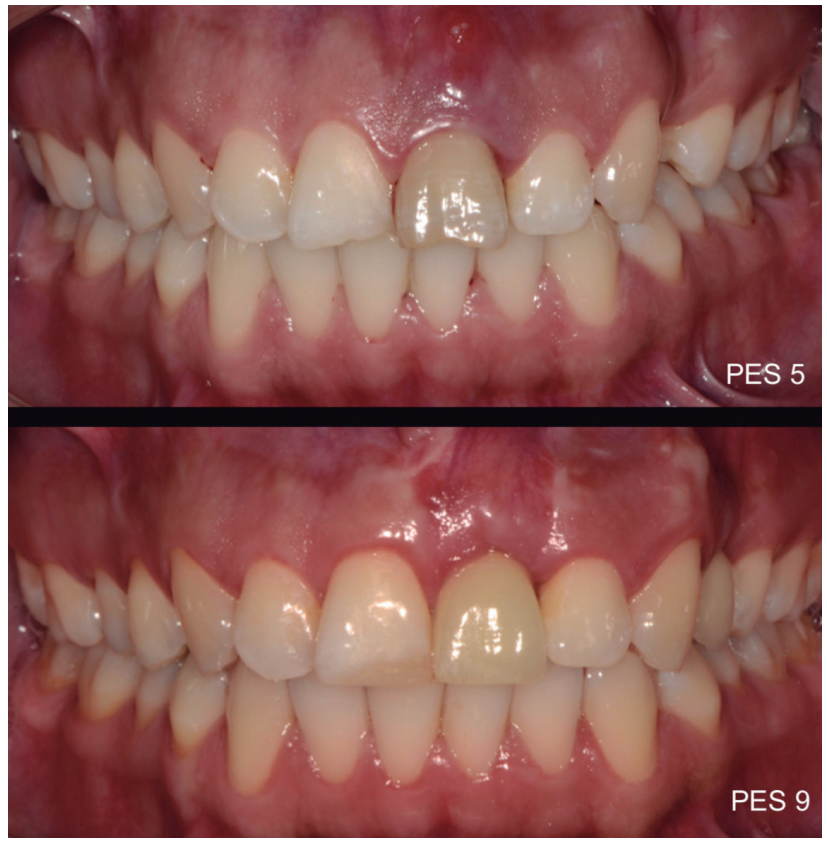

Figura 10.

\section{Bibliografía}

1. Darby I, Chen S, De Poi R. Ridge preservation: what is it and when should it be considered. Aust Dent J. 2008;53(1):11-21.

2. Jivraj S, Chee W. Treatment planning of implants in the aesthetic zone. Br Dent J. 2006;201(2):77-89.

3. Avila-Ortiz G, Chambrone L, Vignoletti F. Effect of alveolar ridge preservation interventions following tooth extraction: a systematic review and meta-analysis. J Clin Periodontol. 2019;46(Suppl 21):195-223.

4. Fürhauser R, Florescu D, Benesch T, Haas R, Mailath G, Watzek G. Evaluation of soft tissue around single-tooth implant crowns: the pink esthetic score. Clin Oral Implants Res. 2005;16(6):639-644.

5. Hurzeler MB, Zuhr O, Schupbach P, Rebele SF, Emmanouilidis N, Fickl S The socket-shield technique: a proof - of -principle report. J Clin Periodontol. 2010;37(9):855-862.

6. Darby I, Chen ST, Buser D. Ridge preservation techniques for implant therapy. Int J Oral Maxillofac Implants. 2009;24(Suppl):260-271.
7. Jung RE, loannidis A, Hämmerle CHF, Thoma DS. Alveolar ridge preservation in the esthetic zone. Periodontol 2000. 2018;77(1):165-175

8. Hämmerle $\mathrm{CH}$, Jung RE. Bone augmentation by means of barrier membranes. Periodontol 2000. 2003;33:36-53.

9.Bassir S, Alhareky M, Wangsrimongkol B, Jia Y, Karimbux N. systematic review and meta-analysis of hard tissue outcomes of alveolar ridge preservation. Int $\mathrm{J}$ Oral Maxillofac Implants. 2018;33(5);979-994.

10. Park JC, Kim CS, Choi SH,Cho Ks, Chai JK, Jung UW. Flap extension attained by vertical and periosteal releasing incision: a prospective cohort study. Clin Oral Implant Res. 2012;23(8):993-998.

11.Tonetti MS, Cortellini P, Graziani F, Cairo F, Lang NP, Abundo R, et al. Immediate versus delayed implant placement after anterior single tooth extraction: the timing randomized controlled clinical trial. J Clin Periodontol. 2017;44(2):215-224. 